\title{
Global Online Interest in Telehealth, Telemedicine, Telerehabilitation, and Related Search Terms amid the COVID-19 Pandemic: An Infodemiological Study
}

\author{
Carl Froilan D. Leochico, PTRP, MD, ${ }^{1,2}$ Edgardo Miguel V. Austria, MD $^{1}$ and Adrian I. Espiritu, MD ${ }^{3,4}$ \\ ${ }^{1}$ Department of Rehabilitation Medicine, College of Medicine and Philippine General Hospital, University of the Philippines Manila, Manila, Philippines \\ ${ }^{2}$ Department of Physical Medicine and Rehabilitation, St. Luke's Medical Center - Quezon City and Global City, Philippines \\ ${ }^{3}$ Department of Clinical Epidemiology, College of Medicine, University of the Philippines Manila, Manila, Philippines \\ ${ }^{4}$ Department of Neurosciences, College of Medicine and Philippine General Hospital, University of the Philippines Manila, Manila, Philippines
}

\begin{abstract}
Background. Catalyzed by the coronavirus disease 2019 (COVID-19) pandemic that restricted non-essential travel, awareness of the value of telehealth increased among healthcare providers and consumers. Telerehabilitation, a subset of telehealth and telemedicine, refers explicitly to the remote provision of rehabilitation services. However, since the majority were new to the concept of telerehabilitation, which was commonly interchanged with other related terms (e.g., telehealth, telemedicine, teletherapy, telecare, telepractice, etc.), and relevant internationally recognized guidelines were not available pre-pandemic, many turned to online resources to learn more about it. However, such might create confusion with the terminologies.
\end{abstract}

Objective. This infodemiological study aimed to examine the trends of global online interest in telehealth, telemedicine, telerehabilitation, and related search terms. The authors hypothesized an abrupt rise in stakeholders' awareness regarding the virtual method of delivering or accessing healthcare, including rehabilitation services, during the pandemic.

Method. Using Google Trends ${ }^{\mathrm{TM}}$, we obtained and analyzed the trends of global online interest in telehealth, telemedicine, telerehabilitation, and other related search terms from 2004 to October 2020.

Results. The frequencies of searches for "telehealth," "telemedicine," and "telerehabilitation" peaked in March 2020, which coincided with the lockdown in many countries, including the Philippines. The term "telehealth" was more frequently searched in western countries, while "telemedicine" was relatively common in eastern countries. Among the terms synonymous with telerehabilitation, "teletherapy" was most commonly used worldwide, with "teletherapy speech" being the most searched related topic. The Philippines had the most significant number of times the term "telerehabilitation" was searched in the past decade.

Conclusion. During the COVID-19 pandemic, there is an overall global demand for the acquisition of knowledge related to telehealth, telemedicine, telerehabilitation, and related search terms. Of particular interest, telerehabilitation, however, varied in terminologies across countries, with "teletherapy" mainly being used worldwide and "telerehabilitation" being primarily searched in the Philippines. Therefore, arriving at a consensus across professional societies and operationally defining different interrelated terms could help streamline our current or future guidelines and programs.

Keywords: Telehealth, telemedicine, telerehabilitation, COVID-19, infodemiology

Corresponding author: Carl Froilan D. Leochico, PTRP, MD

Department of Rehabilitation Medicine

Philippine General Hospital

University of the Philippines Manila

Taft Avenue, Ermita, Manila 1000, Philippines

Email: cdleochico@up.edu.ph 


\section{INTRODUCTION}

In March 2020, the coronavirus disease 2019 (COVID-19) pandemic led the Philippines to go into a quarantine state similar to many others globally. ${ }^{1}$ In the ensuing months, the National Capital Region and other parts of the country have gone through different levels of quarantine to maintain social distancing for health and safety reasons. Nevertheless, the need to maintain a certain level of social distance has given rise to the Internet-Of-Things (IoT) or "tele" era, including telehealth or telemedicine, which became more recognized now compared to the prepandemic period.

Telehealth is commonly interchanged with telemedicine, implying that any healthcare professional can use either term, whether physician or non-physician. In the World Health Organization's (WHO) 2009 report, telemedicine was defined and described as follows:

"Telemedicine, a term coined in the 1970s, which literally means "healing at a distance," signifies the use of information and communications technologies (ICT) to improve patient outcomes by increasing access to care and medical information. Recognizing that there is no one definitive definition of telemedicine, the WHO has adopted the following broad description: delivery of healthcare services, where distance is a critical factor, by all healthcare professionals using ICT for the exchange of valid information for diagnosis, treatment and prevention of disease and injuries, research and evaluation, and for the continuing education of healthcare providers, all in the interests of advancing the bealth of individuals and their communities."

Of particular interest in this article, although telerehabilitation (telerehab), a subset of telehealth and telemedicine, had been present before the pandemic, it has suddenly received greater attention these days. ${ }^{3-8}$ Because this non-traditional approach to rehabilitation has neither been widely known nor implemented in many parts of the world, including the Philippines, telerehab brought about a lot of apprehensions and questions among stakeholders (e.g., patients, clinicians, administrators, educators) regarding its different aspects of implementation and outcomes. ${ }^{9}$ Hence while in quarantine, stakeholders turned to online resources, such as Google ${ }^{\mathrm{TM}}$, for various reasons, such as to feed their curiosity, guide themselves in health-related decisions, and resume healthcare access or delivery. Patients might have searched for telerehabilitation to look for means to continue their consultations and therapy sessions, albeit virtually. At the same time, hospitals remained closed or limited the number of patients that could be accommodated at a time. In parallel, healthcare providers might have been interested in incorporating telerehabilitation into their practice. ${ }^{3-6,8}$

As Google ${ }^{\mathrm{TM}}$ became the most popular search engine possibly because of its efficient indexing and ability to "produce much more satisfying search results" compared to other existing engines, the word "google" was eventually used globally as a verb that means "to search (something)

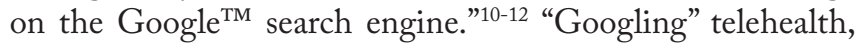
telemedicine, telerehabilitation, and other related or synonymous search terms were suddenly on the rise based on Google Trends ${ }^{\mathrm{TM}}$. This publicly accessible website automatically generates data on "what was and is trending" based solely on Google ${ }^{\mathrm{TM}}$ searches of Internet users worldwide. ${ }^{13}$ Compared to other similar online Web-based applications (e.g., Semrush, Act-On, G2 Storefront, etc.), Google Trends ${ }^{\mathrm{TM}}$ has the following advantages among others: easy to use, free of charge, and quickly provides updated and objective information on search data according to any chosen geographical region and timeframe. ${ }^{14-16}$

However, the search mentioned above terms varied across the globe, especially for telerehabilitation, as there was no standard international operational definition of telerehabilitation. Telerehabilitation has only recently received greater attention, particularly in the Philippines, according to a systematic review. ${ }^{17}$ Before the pandemic, many local rehabilitation providers, were not prepared for the virtual type of technology or service delivery. ${ }^{9}$ Nonetheless, telerehabilitation was proven feasible in clinical and academic settings in different countries, including the Philippines. For instance, since 2017, medical interns rotating in the Department of Rehabilitation Medicine at Philippine General Hospital were already being taught telerehabilitation. ${ }^{18}$ While in the hospital, the students were able to evaluate and manage patients tele-referred from the rural health unit in the province of Alfonso, Cavite. They were able to conduct video-based consultations to manage persons with disabilities and provide local support to the remote community. This form of synchronous telerehabilitation was eventually adopted as part of the teaching-learning activities for medical students from third- to fifth-year levels rotating in the Department. ${ }^{18}$ Meanwhile, local clinical applications of telerehabilitation have already been reported. ${ }^{17}$ The patients and rehabilitation professionals alike have reported telerehabilitation as beneficial and safe. ${ }^{8}$

In general, however, the rehabilitation workforce (i.e., physiatrists or Rehabilitation Medicine physicians; therapists or allied medical professionals) in the clinical and academic settings in public and private sectors needed more information on telerehabilitation apprehensions emerged about its use. ${ }^{17}$ Various sources of information to meet the urgent need for knowledge and skills regarding telerehabilitation became popular in the forms of interaction with colleagues, interim guidelines from international and local professional societies, webinars, and many other online resources based on anecdotal reports. Indeed, acquiring knowledge about it was imperative to prepare for the unprecedented transition from traditional in-person healthcare to virtual means.

Therefore, this infodemiological study aimed to examine the trends of global online interest in telehealth, 
telemedicine, telerehabilitation, and related search terms. The authors hypothesized an abrupt rise in stakeholders' awareness regarding the virtual method of delivering or accessing healthcare, mainly rehabilitation services, during the COVID-19 pandemic. The study results could guide clinicians, educators, and policymakers in operationally defining these key terms to prevent confusion among stakeholders.

\section{METHODS}

"Telehealth" and "telemedicine" were entered as keywords on the main page of Google Trends ${ }^{\mathrm{TM}}$ (available at http:// googletrends.com/trends, accessed October 9, 2020), as telerehabilitation was considered to fall within these umbrella terms. ${ }^{19}$ Google Trends ${ }^{\mathrm{TM}}$ then automatically generated the relative search volume (RSV) of these keywords to assess the changes in interest (i.e., number of searches) in these terms over time. The results from Google Trends ${ }^{\mathrm{TM}}$ were normalized with sets of data divided by a shared variable to cancel out the variable's effects on the data. ${ }^{20}$ The RSV ranged from 0 to 100 , representing the relative proportion a given keyword (e.g., telerehabilitation) was googled over time. ${ }^{15}$ Since RSV was inherently arbitrary and not absolute, normalization was necessary to prevent data from specific geographical locations generating the most extensive search to be consistently ranked highly compared to those with a lesser number of searches. ${ }^{15,20}$ On the other hand, the value zero did not imply that no search at all was done for a particular term, but instead, it indicated a negligible or meager search volume. ${ }^{13}$ The numbers on the graphs reflected how many searches were done for a specific term relative to the number of Google ${ }^{\mathrm{TM}}$ searches over time. Each point in the chart was divided by the highest point, which was conventionally set at $100 .^{20}$

The period of volume search spanned from 2004 (the earliest available coverage of Google Trends ${ }^{\mathrm{TM}}$ ) to October 9, 2020 (the date of data collection). Since "telehealth" and "telemedicine" were commonly interchanged in practice, we compared their percentages of volume search per country. Related search terms were also obtained for "telehealth" and ranked based on their "Rising Interest," as reflected by the number of searches. Google Trends ${ }^{\mathrm{TM}}$ also identified "breakout" term/s for a sudden increase in the number of searches for a particular term over the last 12 months.

Furthermore, the following telerehabilitation-synonymous terms were also entered in Google Trends ${ }^{\mathrm{TM}}$ : "telerehabilitation," "telerehab," "telepractice," "telecare," and "teletherapy." Based on the literature, these terms appeared to be common in the practice of rehabilitation worldwide, varying across disciplines (e.g., physiatry, physical therapy, occupational therapy, speech and language pathology, psychology). The search volume of these terms was examined over two periods: (1) since 2004 to observe long-term trends; and (2) within the past 12 months to depict the shift from pre-pandemic to pandemic periods. In addition, the frequency of search for these terms was obtained for each country within the past year. The top and rising queries related to the five aforementioned telerehabilitationrelated or synonymous terms were also collated and ranked from most to least number of times searched, noting the percentage increase in the number of times a term was searched over a given period. Lastly, the global trend in search frequency for the term "telerehabilitation" was explored in the last ten years, when most advancements in rehabilitation technology most likely occurred.

\section{RESULTS}

Over the last 16 years (from 2004 until February of 2020), there have been relatively lower and more static trends in the number of times the terms "telehealth" and "telemedicine" were searched (Figure 1). There was a significant increase, however, in the online search for "telehealth" and "telemedicine" starting March 2020, with "telehealth" being more commonly searched than "telemedicine" worldwide.

Comparing the frequency of searches for each term per country since 2004, the top 3 countries were Australia, Canada, and New Zealand, which yielded more searches for "telehealth" than "telemedicine" (Figure 2). On the other hand, the United States and the United Kingdom had a relatively equal number of searches for both terms. While for Asian Countries, such as Singapore, Vietnam, and the Philippines, the search term "telemedicine" was used more than "telehealth." Meanwhile, China, India, and France were the top 3 countries that searched for "telemedicine" since 2004.

With regards to the rising terms related to "telehealth" and "telemedicine," there were at least 25 search queries that resulted in an increase in the frequency of searches over the

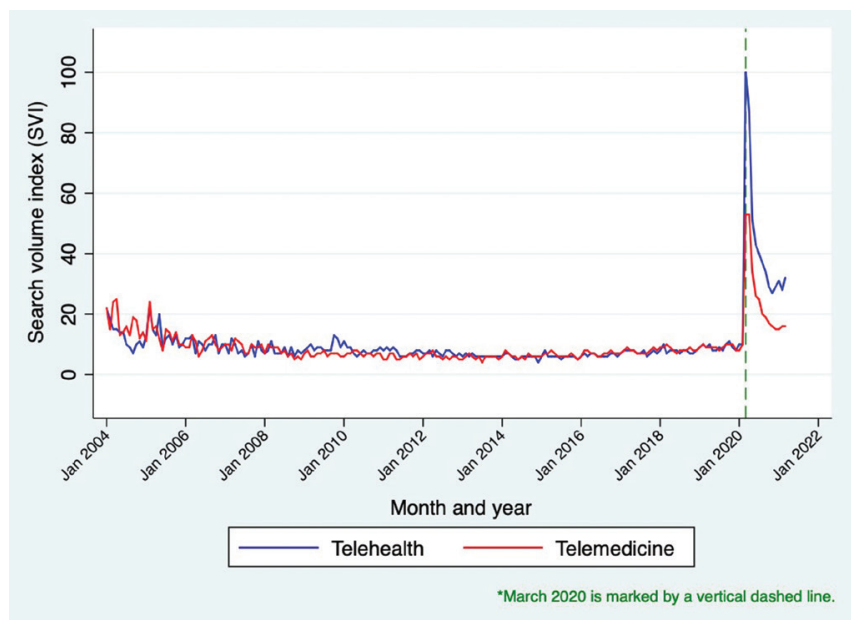

Figure 1. Trends of searches for "telehealth" and "telemedicine" from 2004 (earliest available coverage of Google Trends $^{\mathrm{TM}}$ ) to October 9, 2020 (date of search). 


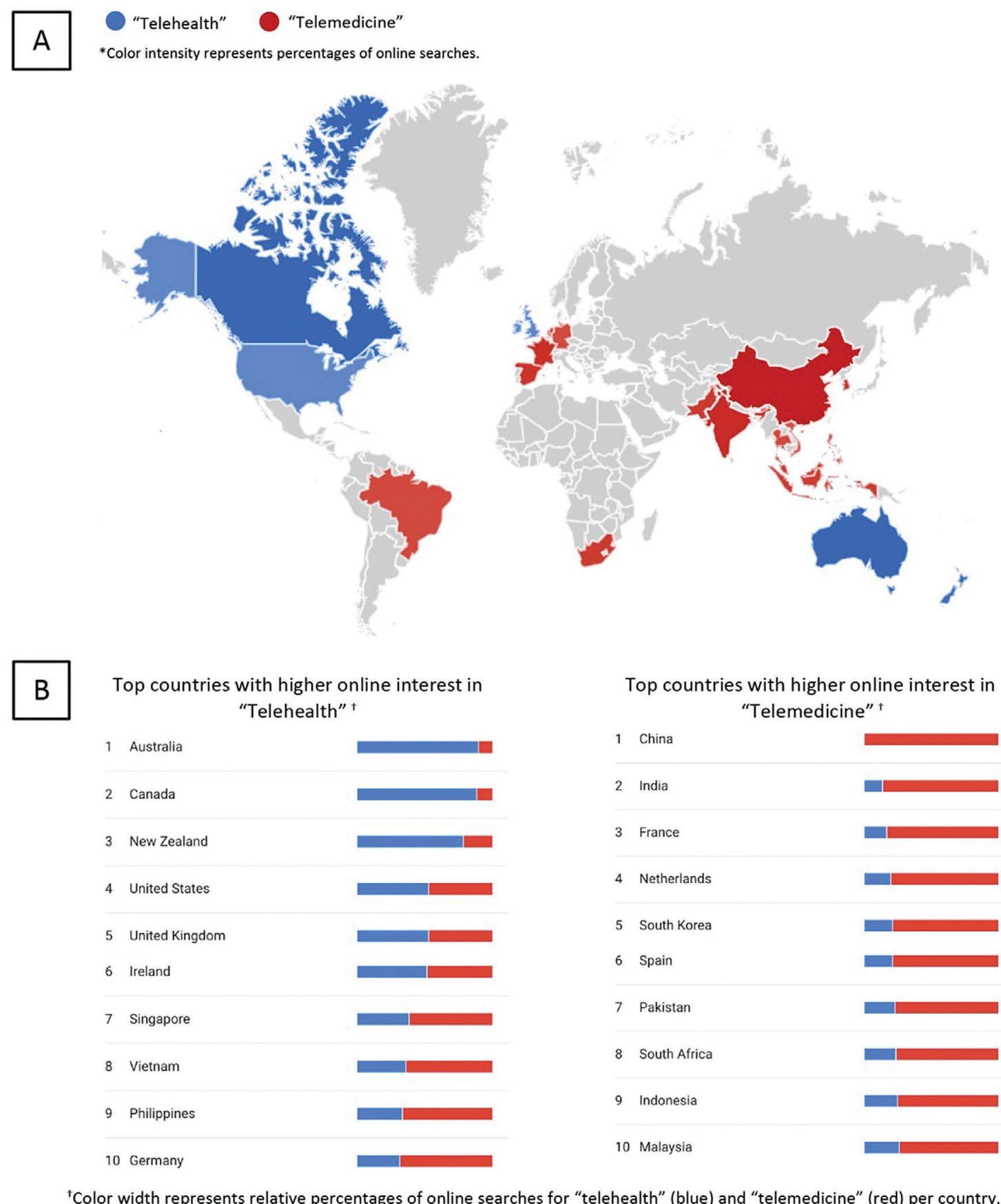

Figure 2. Comparison of searches for "telehealth" and "telemedicine" per countryfrom 2004 to October 9, 2020.

last 12 months (Table 1). When "telehealth" was searched, terms commonly searched too were "telemedicine," "what is telehealth," and "telehealth number," among others, which were considered "rising" terms corresponding to queries with the most significant increase in search frequency relative to the last period. Meanwhile, results marked "breakout" (e.g., "telehealth therapy," "telehealth online," and "mental health telehealth") showed a relative tremendous increase in search frequency, probably because these queries were relatively new and had few (if any) previous searches. ${ }^{20}$
Among the five telerehabilitation-specific terms, "teletherapy" had the highest peak in search frequency around March 2020 (Figure 3). Meanwhile, "telecare" was the most common search term since 2004 until it was superseded by "teletherapy." In general, "telerehabilitation" and "telerehab" were relatively the least commonly used search terms over time.

Regarding the percentage of use of search terms related to telerehabilitation per country, it was noted that "teletherapy" was mainly used worldwide (Figure 4). The top countries 
Table 1. Rising and breakout terms related to "telehealth" worldwide in the past 12 months in decreasing order of frequency

\begin{tabular}{ll}
\multicolumn{1}{c}{ Rising terms } & \multicolumn{1}{c}{ Breakout terms } \\
\hline Telemedicine & Telehealth therapy \\
What is telehealth & Telehealth online \\
Telehealth number & Mental health telehealth \\
Telehealth therapy & Telehealth and telemedicine \\
Home telehealth & Telehealth billing \\
Telehealth phone number & \\
Telehealth mental health & \\
Telehealth and telemedicine & \\
CMS telehealth & \\
Telehealth service & \\
Telehealth billing & \\
CMS & \\
Telehealth definition & \\
Telehealth visit & \\
Ontario telehealth number & \\
Telehealth app & \\
Telehealth codes & \\
VA telehealth & \\
COVID19 telehealth & \\
Telehealth companies & \\
Medicaid telehealth & \\
Telehealth program & \\
Telehealth Australia & \\
Telehealth nurse jobs & \\
Telehealth system &
\end{tabular}

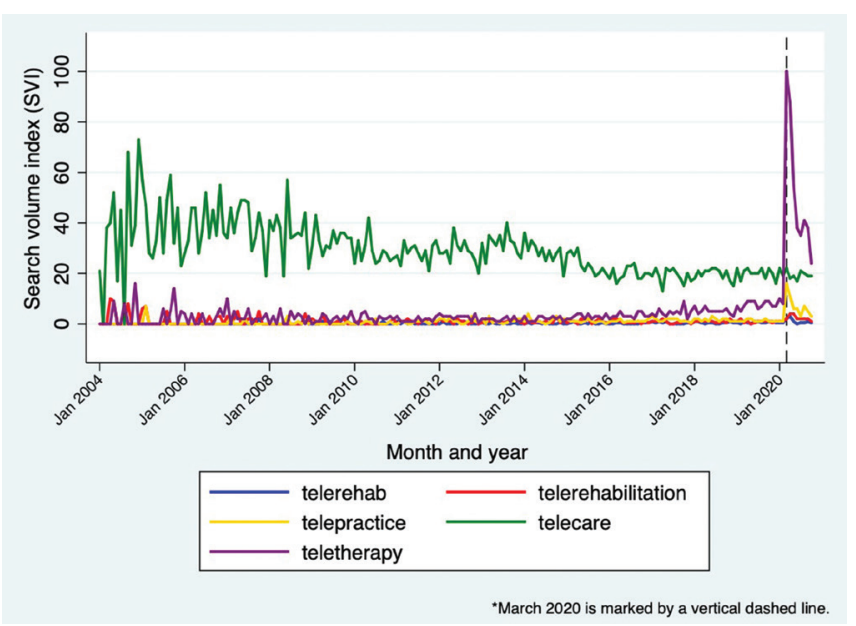

Figure 3. Trends of searches for telerehabilitation-specific terms worldwide from 2004 to October 9, 2020.

used either "telerehabilitation" or "telerehab" as search terms included the Philippines. Compared to "telerehabilitation," "teletherapy" followed by "telecare" seemed to be more commonly used in western countries. While "telepractice" did not yield enough search data (0\%) in the Philippines, "telepractice" was more widely used in Canada, Singapore, and Australia.

When "teletherapy" was used as a search term, terms related to speech and language therapy were the top popular
Table 2. Top and rising terms related to "teletherapy" worldwide in the past 12 months in decreasing order of frequency

\begin{tabular}{ll}
\multicolumn{1}{c}{ Top terms } & \multicolumn{1}{c}{ Rising terms } \\
\hline Teletherapy speech & Teletherapy activities \\
Teletherapy speech therapy & Teletherapy games \\
Teletherapy activities & Asha teletherapy \\
Teletherapy jobs & Boom cards teletherapy \\
Teletherapy games & Teletherapy platforms \\
What is teletherapy & Boom cards \\
Free teletherapy & HIPAA compliant teletherapy \\
Teletherapy occupational therapy & Teletherapy meaning \\
Zoom & Teletherapy Bootcamp \\
Asha teletherapy & Teletherapy games for kids \\
Speech teletherapy jobs & Teachers pay teachers \\
Teletherapy mental health & Teletherapy across state lines \\
Boom cards teletherapy & Teletherapy activities for kids \\
Teletherapy platforms & OT teletherapy ideas \\
Boom cards & Benefits of teletherapy \\
Global teletherapy & Abcya \\
HIPAA compliant teletherapy & Zoom \\
SLP teletherapy jobs & Teletherapy speech \\
Teletherapy meaning & Free teletherapy \\
Teletherapy definition & Teletherapy speech therapy \\
Teletherapy Bootcamp & Teletherapy occupational therapy \\
Teletherapy games for kids & What is teletherapy \\
Teachers pay teachers & \\
Teletherapy across state lines & \\
Teletherapy activities for kids & \\
\hline
\end{tabular}

search queries worldwide, ranking first and second in the last 12 months (Table 2). Meanwhile, the rising terms were found to be "teletherapy activities" and "teletherapy games."

Looking into the global trend of search frequency for the term "telerehabilitation" over the past ten years, it was noted that the Philippines showed the highest online interest (Figure 5).

\section{DISCUSSION}

This infodemiological study confirmed our hypothesis that there was an abrupt rise in the interest in telehealth, telemedicine, and telerehabilitation based on online search behavior using Google Trend ${ }^{\mathrm{TM}}$. The term "telemedicine" was more commonly searched than "telehealth" in the Philippines and other eastern countries. Among the telerehabilitationspecific terms, "teletherapy" was mainly searched with the most significant association with speech and language therapy services.

Gunther Eysenbach defined "infodemiology" as "the science of distribution and determinants of information in an electronic medium, specifically the Internet, or in a population, with the ultimate aim to inform public health and public policy." ${ }^{21}$ Infodemiology identifies areas wherein there is a knowledge translation gap between best evidence (what some experts know) and practice (what most people do or believe), as well as markers for "high quality" information. ${ }^{21}$ Through this infodemiological study, we 

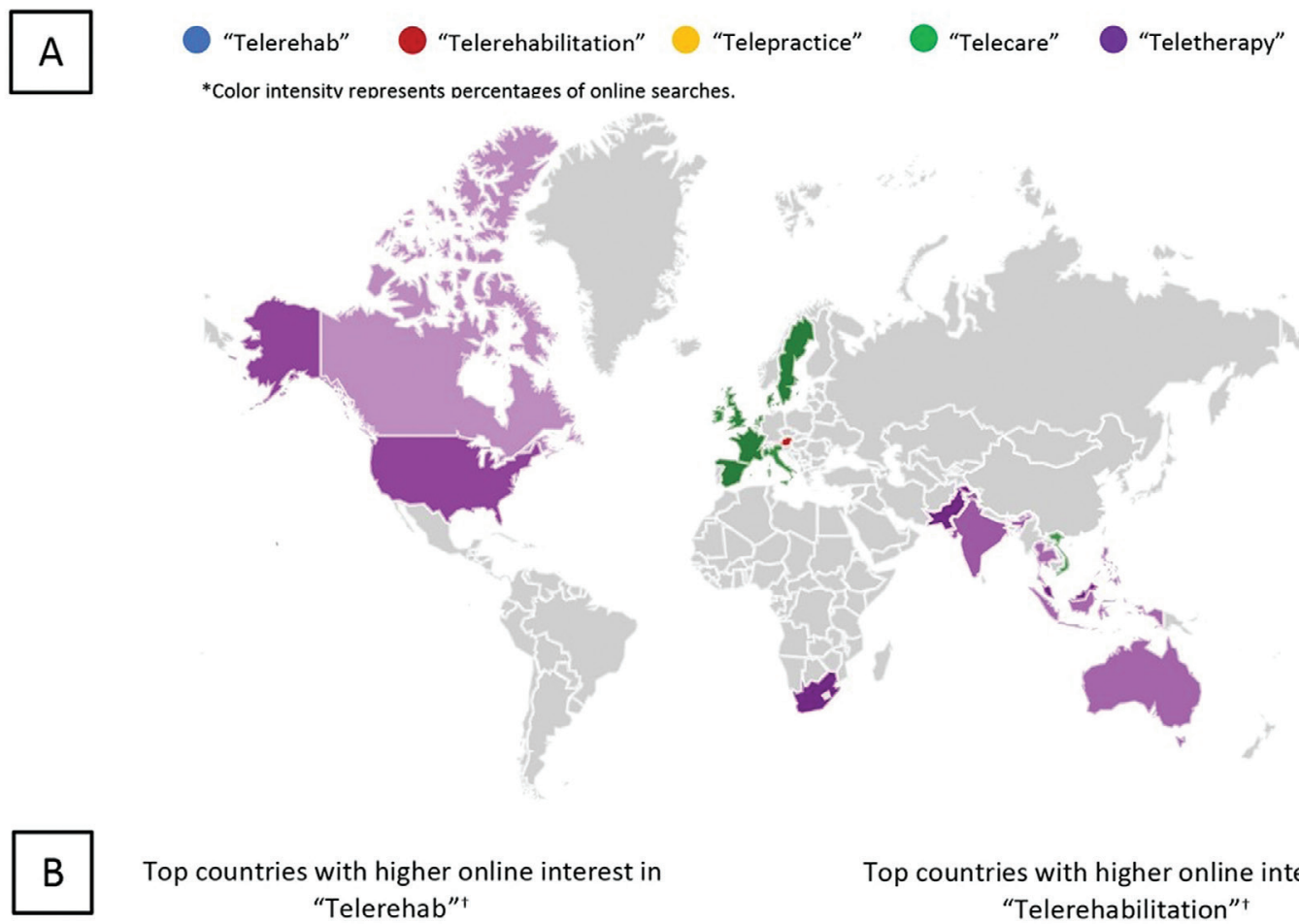

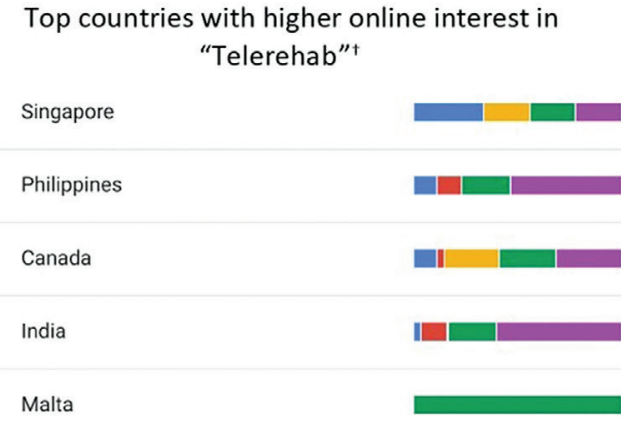

Top countries with higher online interest in "Telepractice" ${ }^{+}$

1 Canada
2 Singapore
3 Australia
4 United States
5 Ireland

Top countries with higher online interest in "Teletherapy"

$\begin{array}{ll}1 & \text { South Africa } \\ 2 & \text { Pakistan } \\ 3 & \text { Malaysia } \\ 4 & \text { United States } \\ 5 & \text { India }\end{array}$

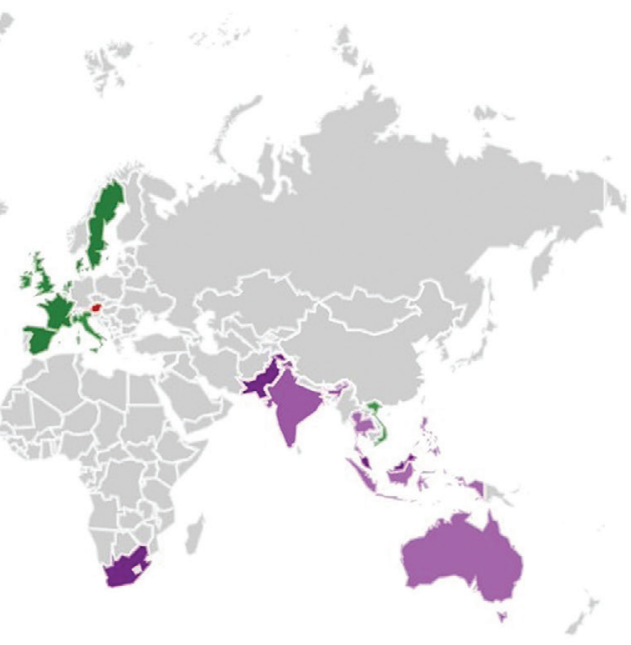

Top countries with higher online interest in "Telerehabilitation" ${ }^{+}$

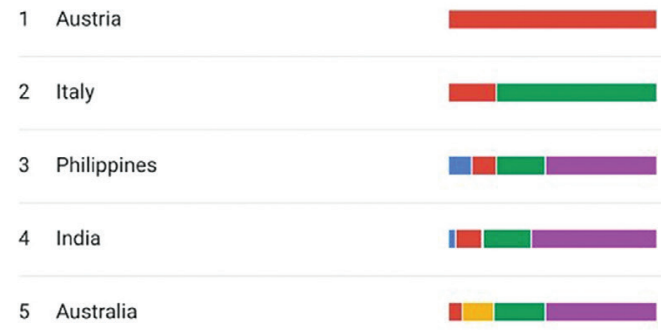

Top countries with higher online interest in "Telecare" ${ }^{+}$

$\begin{array}{ll}1 & \text { Malta } \\ 2 & \text { St Helena } \\ 3 & \text { Sweden } \\ 4 & \text { Netherlands } \\ 5 & \text { Denmark }\end{array}$

${ }^{+}$Color width represents relative percentages of online searches for "telerehab" (blue), "telerehabilitation" (red), "telepractice" (yellow), "telecare" (green), "teletherapy" (purple) per country.

Figure 4. Comparison of searches for telerehabilitation-specific terms per country in the past 12 months. 


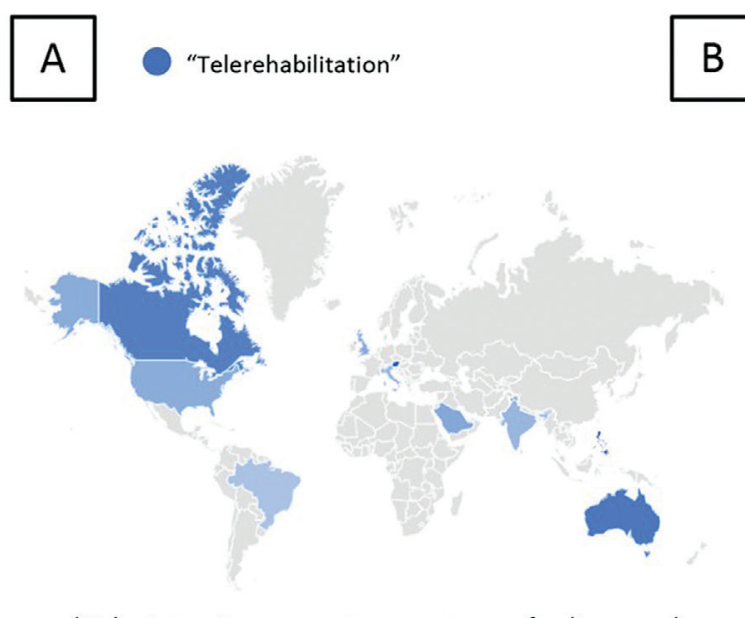

*Color intensity represents percentages of online searches.
Top countries with higher online interest in

"Telerehabilitation"

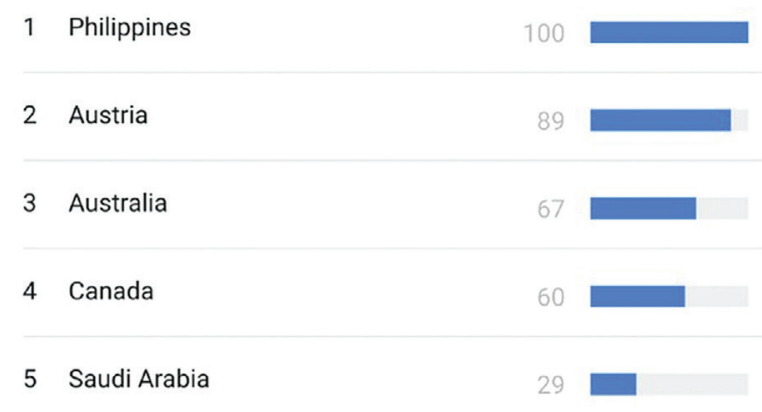

${ }^{\dagger}$ Color width represents percentages of online searches for "telerehabilitation" (blue).

Figure 5. Search frequencies for "telerehabilitation" by country in the past ten years.

were able to document the sudden increase in the rise of interest in telehealth, telemedicine, and telerehabilitation on a global scale, peaking in March 2020. This peak coincided with the declaration of a pandemic by the $\mathrm{WHO}$ and the subsequent implementation of lockdown or quarantine in various regions worldwide, including the Philippines. ${ }^{1,22}$ In addition, we were also able to determine the variations of terms related to digital delivery of healthcare and rehabilitation services used by the majority of populations across the globe.

Since "telemedicine" and "telehealth" were commonly interchanged in practice, we compared the percentages of volume searches of these two keywords per country. Search term popularity was proportional to the total number of Google ${ }^{\mathrm{TM}}$ searches performed at a specific time within a particular location. Based on the results, "telehealth" seemed to be more commonly used in western countries, wherein such service had been implemented for a more extended period. At the same time, "telemedicine" was more common in the eastern part of the world that might be relatively new to the "tele" services in healthcare. A reasonable basis for the difference between terms used across countries might be in their respective national laws, guidelines, and/ or organizations, if any, whether they used telehealth or telemedicine. The popular culture at that time when the search was made in a particular country was another possible explanation for the differences in search terms. Nonetheless, it was appropriate that the WHO clarified these terms and considered them synonymous to avoid confusion, especially with the misconception that physicians could only provide telemedicine. ${ }^{2}$ However, looking at it from a different angle, the other schools of thought have also made proper distinctions between "telehealth" and "telemedicine" as to who can practice them. Consistent with the Philippine Medical Act of 1959, any physician can practice telemedicine with a valid medical license. ${ }^{23}$ Meanwhile, according to the Philippine Telehealth Act of 2014, telehealth can be practiced by any registered healthcare professional, whether a medical or paramedical provider, within the scope of his/her professional license. ${ }^{24}$ The differences between the aforementioned schools of thought (i.e., WHO and Philippine policies) might be operational definitions and cultural adaptations that have to be simply clarified among stakeholders to prevent confusion and increase consistency and adoption.

It was found in this study that most of the terms associated with the search queries for "telehealth" and "telemedicine" were related to finding out what services telehealth and telemedicine could provide and who to contact to avail of these services. Billing for telehealth and virtual mental health programs were also among the top related searches. These provide information on the kind of data that stakeholders were generally interested to know more about. For instance, it could be inferred that the healthcare providers and consumers (e.g., patients, family, caregivers) alike were curious about acceptable professional fees and payment methods, given that a lot of them might not have tried this virtual method of healthcare services before.

With this, the term "teletherapy" was also noted to have peaked within the same period that the volume of search queries for "telehealth" and "telemedicine" also increased. Among the terms synonymous with "teletherapy," "teletherapy speech" received the highest percentage of searches. It seemed that speech therapy through "tele" has always been the most popular compared to other therapy services (e.g., physical therapy, occupational therapy, etc.). Unlike physical therapy and occupational therapy that frequently required handson or manual assistance for treatment effectiveness and safety, speech therapy and even psychology could be quickly done by videoconferencing. ${ }^{25}$ It might be true that speech therapists have been relatively more skilled and experienced in conducting teletherapy even before the pandemic, as many aspects in their practice could be safely and practically administered even from a distance. Likewise, clients of speech 
therapists might have also experienced or realized firsthand the benefits of speech teletherapy compared to other therapy services (e.g., physical or occupational therapy).

The sudden interest in improving one's knowledge on telerehabilitation or teletherapy coincided with the surge of cases of COVID-19 worldwide. Due to the infection risks that accompanied traditional or in-person rehabilitation, many stakeholders (e.g., patients, doctors, therapists, academicians) became open to the concept of virtual methods, such as telerehabilitation. Global organizations, such as the WHO, have been advocating telehealth for several years, even pre-pandemic, to address critical health challenges, especially in low-income countries in Asia, Africa, and Latin America. ${ }^{2}$ Although the progress of telehealth endeavors varied from region to region across the world, it was inevitable that the COVID-19 pandemic had catalyzed this shift in healthcare delivery. Despite this, implementing telemedicine or telehealth was no easy feat. Awareness of telemedicine was not enough, especially since most healthcare professionals were caught unprepared or untrained for virtual care. Using telehealth requires developing new skills not taught in formal undergraduate and graduate academic or training institutions. ${ }^{9,26}$ In particular, academic and training curricula must be revisited to prepare future clinicians not just for similar unprecedented circumstances but also for the new normal. New competencies in telehealth, particularly telerehabilitation or teletherapy, should be honed, reinforced, and supported through proper training and education of current and future rehabilitation providers. ${ }^{9}$

This study documented the various telerehabilitation terms useful in policy-making and academic or service program planning and implementation. However, knowing that the Philippines ranked first worldwide in the interest in this relatively new rehabilitation technique, it might be safe to assume that various stakeholders were hungry for more reliable information about it. Currently, there was minimal local literature on telerehabilitation, reflecting the widespread lack of experience in conducting telerehabilitation throughout the country, possibly similar to other countries just beginning to adopt "tele" in their practice. Lack of knowledge, skills, and experiences in virtual care might pose an imminent threat to patient safety, data privacy, ethics, and legalities. Moreover, pre-pandemic telehealth, more so telerehabilitation, had never been widely or formally included in the expected learning outcomes for students in medical schools and rehabilitation science colleges worldwide. Locally, however, it was of note that since 2017 the Department of Rehabilitation Medicine at Philippine General Hospital, University of the Philippines Manila had been teaching telerehabilitation principles, simulation, and actual practice to medical students during their rotations in Learning Units 5 (i.e., 3rd-year level), 6 (i.e., 4th-year level or clinical clerkship), and 7 (i.e., 5th-year level or clinical internship), respectively. ${ }^{27}$ Such experience in telerehabilitation had prepared the Department and hopefully its past students for the pandemic.
Furthermore, the term telerehabilitation seemed to have been made popular locally by the University of the Philippines Manila, which might be considered early adopters, if not the forerunners, of telerehabilitation. Their relatively more expansive and growing experience with telerehabilitation in terms of service, training, and research might have catalyzed the increased awareness regarding "telerehabilitation" or "telerehab" among stakeholders, whether patients, therapists, or other specialists, throughout the country. ${ }^{8}$ In this digital age, the word could have easily spread through local and international webinars and social media, especially during the COVID-19 pandemic, when telerehabilitation became suddenly more relevant than in previous years.

As more and more rehabilitation professionals in the academe, training, and service became more interested in adopting telerehabilitation for their respective stakeholders, a need for a more widely accepted national policy should be in place to guide and standardize practice. The uncertainty about which telerehabilitation-specific term was appropriate to use might be due to lack of internationally recognized standard or guideline on telerehabilitation in the background of varying practices, needs, resources, set-ups, skills, and technological capacities across healthcare settings. A blueprint of telerehabilitation guideline by Brennan and colleagues, however, suggested to include the following contents: scope and definitions, guiding principles, clinical principles, technical principles, and ethical principles. ${ }^{28}$

This infodemiological study provided evidence that the term "teletherapy" was more commonly searched or probably used than "telerehabilitation" in the Philippines. The relatively greater search for the term "teletherapy" could be attributed to at least three factors: (1) the more significant number of allied rehabilitation professionals compared to Rehabilitation Medicine physicians in the Philippines who could have searched the term $;^{17}(2)$ the term "teletherapy" in the field of Radiology could have been searched as well by patients and radiologists interested in external beam radiotherapy, ${ }^{29}$ rather than rehabilitative, therapeutic services, and (3) the term "teletherapy" used in the field of Psychiatry. ${ }^{30}$ Such clarifications in terminology could be considered when updating the interim telerehabilitation guidelines of the Philippine Academy of Rehabilitation Medicine and other local paramedical professional societies. Of important note, telerehabilitation is relatively young in the field of Physical Medicine and Rehabilitation worldwide. It has only been studied within the past 20 years, and up to now, there seems to be no international or standard agreement on the definition of telerehabilitation. ${ }^{31}$ Unlike other specialties that solely utilized teleconsultations, "telerehabilitation" in the practice of Rehabilitation Medicine should include both "teleconsultations" and "teletherapy" in the treatment armamentarium of physiatrists and other rehabilitation professionals. Hence, there should be a culturally-adapted distinction between these two terms during policy- or guideline-making at least applicable in our local healthcare 
setting, such as in terms of, but not limited to, the following: standard definitions, scope or coverage of services, and who could provide the service based on qualifications.

The limitations of our study were inherently related to the Google Trends ${ }^{\mathrm{TM}}$ algorithm, which solely relies on data entered in the Google ${ }^{\mathrm{TM}}$ search engine. Hence, the data gathered in this study might have missed several relevant searches made using other search engines, including the scholarly databases like PubMed ${ }^{\mathrm{TM}}$, commonly used by healthcare professionals across clinical, academic, and research settings. Using Google Trends ${ }^{\mathrm{TM}}$ also provided us information on mere relative search values (i.e., normalized indexes) rather than exact search volumes, limiting our data analysis. ${ }^{32}$ Furthermore, our search only used English terms. Some multi-lingual countries or multiple dialects might have used other search terms with similar meanings to the terms we used in this study. These translated terms entered in Google ${ }^{\mathrm{TM}}$ were not accounted for in the present study. Another limitation inherent to the data collection platform was the inability to classify the stakeholders according to patients or healthcare providers who previously searched for the terms. Such information could have been helpful, especially in planning telerehabilitation activities or programs unique for each stakeholder category. Although the use of Google Trends ${ }^{\mathrm{TM}}$ in epidemiologic studies had increased in recent years, the reliability of the information it could provide could be considered speculative. ${ }^{15}$ Further research might be necessary to standardize its use and improve its accuracy. ${ }^{13}$

Regardless of these limitations, our study provided interesting data supporting the growing need for knowledge on remote access to healthcare or rehabilitation. We hope that this study would serve as a reference for future research endeavors in the field of telehealth, telemedicine, or telerehabilitation in the Philippines, as well as other countries beginning to shift to virtual rehabilitation care. It could be acknowledged that the fields of telehealth, telemedicine, telerehabilitation, and other related terms have been growing. However, amid their diverse and dynamic operational definitions, their end goals would most likely remain the same: providing health-related services over a distance by leveraging information and communications technologies to overcome the barriers to in-person healthcare.

\section{CONCLUSION}

The pandemic seemed to have catalyzed the increased interest and awareness of healthcare providers and consumers regarding the potential of remote or virtual access to healthcare and rehabilitation services, regardless of terms used (i.e., telehealth, telemedicine, telerehabilitation, and other related search terms). Of particular interest, telerehabilitation varied in terminologies across countries, with "teletherapy" mainly being used worldwide and "telerehabilitation" mostly being searched in the Philippines. Arriving at a consensus across professional societies and operationally defining various interrelated terms could help streamline our current or future guidelines and programs. Further research is recommended to gather and analyze data similar to the present study, but including online search behavior for terms entered in other existing engines or scholarly databases, where possible.

\section{Ethical considerations}

This study did not require ethical board review since it only utilized published aggregate data from Google Trends ${ }^{\mathrm{TM}}$. No patient information was presented in this study.

\section{Statement of Authorship}

CFDL: Conceptualization, data curation, formal analysis, interpretation of data, writing-original draft, writing-review, and editing. EMVA: Conceptualization, data curation, formal analysis, interpretation of data, writing-original draft, writing-review, and editing. AIE: Conceptualization, data curation, formal analysis, interpretation of data, writingoriginal draft, writing-review, and editing.

\section{Author Disclosure}

All authors declared no conflicts of interest.

\section{Funding source}

This study did not receive any specific grant from funding agencies in the public, commercial, or not-for-profit sectors.

\section{REFERENCES}

1. Office of the President of the Philippines Malacañang. Community quarantine over the entire Luzon and further guidelines for the management of the coronavirus disease 2019 (COVID-19) situation: Memorandum from the Executive Secretary [Internet]. 2020 [cited 2020 Mar 20]; Available from: https://www.officialgazette.gov. $\mathrm{ph} /$ downloads/2020/03mar/20200316-MEMORANDUM-FROMES-RRD.pdf.

2. World Health Organization (WHO). Telemedicine: Opportunities and Developments in Member States: Report on the Second Global Survey on e-health. Global observatory for e-health series - volume 2:8-9. NLM classification W 26.5. ISBN 9789241564144. ISSN 2220-5462. Geneva: 2010.

3. Scherrenberg M, Wilhelm M, Hansen D, Völler H, Cornelissen V, Frederix I, et al. The Future is Now: A Call for Action for Cardiac Telerehabilitation in the COVID-19 Pandemic from the Secondary Prevention and Rehabilitation Section of the European Association of Preventive Cardiology. Eur J Prev Cardiol [Internet] 2020;0(0): 1-21. [cited 2021 Apr 7]; Available from: https://academic.oup.com/ eurjpc/advance-article/doi/10.1177/2047487320939671/6145732.

4. Chang MC, Boudier-Revéret M. Usefulness of Telerehabilitation for Stroke Patients During the COVID-19 Pandemic. Am J Phys Med Rehabil [Internet] 2020;99(7):582-582. [cited 2021 Apr 7]; Available from: https://journals.lww.com/10.1097/PHM.0000000000001468.

5. Pugliese M, Wolff A. The Value of Communication, Education, and Self-management in Providing Guideline-based Care: Lessons Learned from Musculoskeletal Telerehabilitation During the COVID-19 Crisis. HSS J ${ }^{\circledR}$ [Internet] 2020;16(S1):160-3. [cited 2021 Apr 7]; Available from: http://journals.sagepub.com/doi/10.1007/ s11420-020-09784-2.

6. Pinto M, Gimigliano F, De Simone S, Costa M, Bianchi AAM, Iolascon G. Post-acute Covid-19 Rehabilitation Network Proposal: 
From Intensive to Extensive and Home-based it supported services. Int J Environ Res Public Health 2020;17(24):1-14. [cited 2021 Apr 7]; Available from: https://doi.org/10.3390/ijerph17249335.

7. Niknejad N, Ismail W, Bahari M, Nazari B. Understanding Telerehabilitation Technology to Evaluate Stakeholders' Adoption of Telerehabilitation Services: A Systematic Literature Review and Directions for Further Research. Arch Phys Med Rehabil [Internet] 2021 (Article in Press). [cited 2021 Apr 7]; Available from: https:// doi.org/10.1016/j.apmr.2020.12.014.

8. Leochico CFD, Mojica JAP, Rey-Matias RR, Supnet IE, Ignacio SD. Role of Telerehabilitation in the Rehabilitation Medicine Training Program of a COVID-19 Referral Center in a Developing Country. Am J Phys Med Rehabil [Internet] 2021; (Article in Press). [cited 2021 Apr 7]; Available from: https://journals.lww.com/10.1097/ PHM.0000000000001755.

9. Leochico CFD. Adoption of Telerehabilitation in a Developing Country before and during the COVID-19 Pandemic. Ann Phys Rehabil Med [Internet] 2020. [cited 2020 Sep 2]; Available from: https://linkinghub.elsevier.com/retrieve/pii/S1877065720301238.

10. Brin S, Page L. The Anatomy of a Large-scale Hypertextual Web Search Engine. Comput Networks ISDN Syst [Internet] 1998;30(17):107-17. Available from: https://linkinghub.elsevier.com/retrieve/ pii/S016975529800110X.

11. Ziakis C, Vlachopoulou M, Kyrkoudis T, Karagkiozidou M. Important Factors for Improving Google Search Rank. Futur Internet [Internet] 2019;11(2):32. Available from: http://www.mdpi.com/1999$5903 / 11 / 2 / 32$.

12. Harper D. Online Etymology Dictionary: Google (v.) [Internet]. n.d. [cited 2021 Jun 12];Available from: https://www.etymonline.com/ word/google.

13. Mavragani A, Ochoa G. Google Trends in Infodemiology and Infoveillance: Methodology Framework. JMIR Public Heal Surveill [Internet] 2019;5(2):e13439. Available from: http://publichealth.jmir. org/2019/2/e13439/.

14. Jun S-P, Yoo HS, Choi S. Ten Years of Research Change Using Google Trends: from the Perspective of Big Data Utilizations and Applications. Technol Forecast Soc Change [Internet] 2018;130: 69-87. Available from: https://linkinghub.elsevier.com/retrieve/pii/ S0040162517315536.

15. Cervellin G, Comelli I, Lippi G. Is Google Trends a Reliable Tool for Digital Epidemiology? Insights from Different Clinical Settings. J Epidemiol Glob Health [Internet] 2017;7(3):185. Available from: https://www.atlantis-press.com/article/125905822.

16. G2 Company. Google Trends Alternatives \& Competitors [Internet]. n.d. [cited 2021 Jun 12];Available from: https://www.g2.com/products/ google-trends/competitors/alternatives.

17. Leochico C, Espiritu A, Ignacio S, Mojica J. Challenges to the Emergence of Telerehabilitation in a Developing Country: A Systematic Review. Front Neurol. 2020;11:1007.

18. Leochico CF, Mojica JA. Telerehabilitation as a Teaching-learning Tool for Medical Interns. PARM Proc. 2017;9(1):39-43.

19. Schmeler MR, Schein RM, McCue M, Betz K. Telerehabilitation and Clinical Applications: Research, Opportunities, and Challenges. Int J Telerehabil [Internet] 2015;(Supplemental Issue):12-24. [cited 2020 Sep 2]; Available from: https://telerehab.pithttps//telerehab.pitt. edu/ojs/index.php/Telerehab/article/view/701/951t.edu/ojs/index. $\mathrm{php} /$ Telerehab/article/view/701/951.
20. Brigo F, Igwe SC, Ausserer H, Nardone R, Tezzon F, Bongiovanni LG, et al. Why Do People Google Epilepsy? Epilepsy Behav [Internet] 2014;31:67-70. [cited 2020 Sep 2]; Available from: https:// linkinghub.elsevier.com/retrieve/pii/S1525505013006185.

21. Eysenbach G. Infodemiology and Infoveillance: Framework for an Emerging Set of Public Health Informatics Methods to Analyze Search, Communication and Publication Behavior on the Internet. J Med Internet Res [Internet] 2009;11(1):e11. [cited 2020 Sep 2]; Available from: http://www.jmir.org/2009/1/e11.

22. Ducharme J. World Health Organization Declares COVID-19 A "Pandemic:" Here's What That Means [Internet]. Time [Internet]. [cited 2020 Nov 16]; Available from: https://time.com/5791661/whocoronavirus-pandemic-declaration/.

23. The Senate and The House of Representatives of the Congress of the Philippines. Republic act no. 2382, The Medical Act of 1959. Philippines: 1959 [Internet]. [cited 2021 Apr 6]; Available from: https://www.prc.gov.ph/uploaded/documents/MEDICAL\%20 LAW.pdf.

24. The House of Representatives of the Congress of the Philippines. House Bill No. 4199, The Telehealth Act of 2014. Manila, Philippines: Sixteenth Congress, First Session; 2014 [Internet]. [cited 2021 Apr 6]; Available from: https://www.congress.gov.ph/legisdocs/basic_16/ HB04199.pdf.

25. Pramuka M, Van Roosmalen L. Telerehabilitation Technologies: Accessibility and Usability. Int J Telerehabilitation [Internet] 2009;1(1):85-98. [cited 2020 Nov 28]; Available from: http://telerehab. pitt.edu/ojs/index.php/Telerehab/article/view/6016.

26. Edirippulige S, Armfield N. Education and Training to Support The Use of Clinical Telehealth: A Review of the Literature. J Telemed Telecare [Internet] 2017;23(2):273-82. [cited 2020 Oct 20]; Available from: http://journals.sagepub.com/doi/10.1177/1357633X16632968.

27. Supnet I, Mojica JA, Ignacio S, Leochico CF. Development of an Evaluation Tool for The Assessment of Telemedicine Courses. Asia Pacific Sch. Forthcoming 2021.

28. Brennan D, Tindall L, Theodoros D, Brown J, Campbell M, Christiana D, et al. A Blueprint for Telerehabilitation Guidelines. Int J Telerehabilitation [Internet] 2010;2(2):31-4. [cited 2020 Dec 8]; Available from: http://telerehab.pitt.edu/ojs/index.php/Telerehab/ article/view/6063.

29. Pereira GC, Traughber M, Muzic RF. The Role of Imaging in Radiation Therapy Planning: Past, Present, and Future. Biomed Res Int [Internet] 2014;2014:1-9. [cited 2021 Apr 6]; Available from: http://www.hindawi.com/journals/bmri/2014/231090/.

30. Markowitz JC, Milrod B, Heckman TG, Bergman M, Amsalem D, Zalman H, et al. Psychotherapy at a Distance. Am J Psychiatry [Internet] 2021;178(3):240-6. [cited 2021 Apr 6]; Available from: http://ajp.psychiatryonline.org/doi/10.1176/appi.ajp.2020.20050557.

31. Jafni TI, Bahari M, Ismail W, Radman A. Understanding the Implementation of Telerehabilitation at Pre-implementation Stage: A Systematic Literature Review. Procedia Comput Sci [Internet] 2017;124:452-60. [cited 2021 Apr 6]; Available from: https:// linkinghub.elsevier.com/retrieve/pii/S1877050917329459.

32. Layug EJV, Espiritu AI, Calotes-Castillo LV, Jamora RDG. The association of online search interest with polio cases and vaccine coverage: an infodemiological and ecological study. Eur J Pediatr 2021 [published ahead of print]. Available from https://doi.org/10.1007/ s00431-021-04049-4. 